\title{
Research on Insertion Process of Medical Needle
}

\author{
Lelun Jiang ${ }^{1 \mathrm{a}}$, Yong Huang ${ }^{2 \mathrm{~b}}$, Chengfeng Pan ${ }^{1 \mathrm{c}}$, Jintian Ling ${ }^{1}$, Jiyu $\mathrm{Li}^{1}$, Zhipeng \\ Chen $^{1}$, Jilin $\mathrm{Fu}^{2}$, Yubiao Wen ${ }^{2}$ \\ ${ }^{1}$ Guangdong Provincial Key Laboratory of Sensor Technology and Biomedical Instrument, Sun \\ Yat-sen University, Guangzhou 510006, China; \\ ${ }^{2}$ Guangdong Medical Devices Quality Surveillance and Test Institute, Guangzhou 510663, China. \\ ajianglel@mail.sysu.edu.cn, ${ }^{b}$ huangyong821@163.com, ${ }^{c}$ panchf@mail2.sysu.edu.cn
}

Keywords: Loading equipment; Medical needle; Insertion force; FEM

\begin{abstract}
This Medical needle plays an important role in modern clinical medicine. The penetration and pull process of medical injection needle was simulated by FEM software Abaqus, and the equivalent stress and strain were analyzed. Self-developed mechanical loading equipment was adopted to test the penetration force with different needle tip angles, needle diameters and penetration speeds. The results showed that: the puncture of medical needle into skin was mode I crack. The maximum stress and strain was focused on skin around the tip of needle. The penetration force decreased with needle diameter and penetration speed. The angle of needle tip form greatly affected the penetration force. When the angle of needle tip was $90^{\circ}$, the penetration force reached a maximum value of $708.3 \mathrm{mN}$, which was 2.21 times that of the needle tip angle $30^{\circ}$. The penetration force of isosceles triangle tip was minimum value.
\end{abstract}

\section{Introduction}

The syringe is always applied in subcutaneous injection drugs, blood sampling and local anesthesia and so on [1]. Injections without pain or with mild pain can allay psychological fear of patients. One reason of injection pain is caused by large resistance between the needle and human skin. The needle stimulates skin and causes tissue contraction. The greater the stimulation intensifies, the more the tissue shrinks, and the stronger one feels painful. Skin cells were deformed or destroyed with the increase of resistance between skin and the needle during the penetration process. The skin cells released induced pain substances, such as potassium ions, hydrogen ions, plasma kinin and so on [2]. Penetration and pull force are affected by the needle size, shape, and puncture speed. Research on penetration and pull force of injection needle with the goal of reducing pain may provide an inspiration for the further design of improved medical injection needles.

Researches on penetration properties of tissue have been reported. Podder et al. [3] measured the mechanical properties of a solid needle puncturing tissues. O'Leary et al. [4] studied puncture stress-strain relation of solid needle with different sizes. Saito et al. [5] and Aoyagi et al. [6] found that puncture force could decrease by the sharpening of needle tip. DiMaio et al. [7] analyzed the relationship between needle insertion force and displacement and established two-dimensional linear elastic FEM. Qi Yingchun et al. [8] reduced stimulation of pain nerve, improved the syringe needle shape, reduced the friction between needle and soft tissue, but it failed to research the impact of the injection needle puncture speed on the puncture resistance. Maurin et al. [9] found the relationship between axial force and penetration displacement depth in the surgery needle penetration and withdrawing process, researched penetration resistance of needle puncturing living liver, but the research did not discuss penetration resistance at different needle tip shapes. Therefore, research on mechanical properties of needle insertion provides theoretical basis for better design of medical needle. However, above researches did not consider the impact of the puncture speed and skin tissue friction properties on penetration force.

The purpose of this paper is to research on puncture and pull force of medical needle during injection and optimize needle shape and puncture style. We would study on the puncture mechanical 
properties of medical needle at different diameters, tip angles and speeds. Abaqus FEM would be established to simulate medical needle injection process. The research may provide an inspiration for the further design of improved medical needle.

\section{Experimental}

\subsection{Material Preparation.}

Human skin was difficult to get and its mechanical properties were greatly affected by temperature, age, humidity, $\mathrm{PH}$ and so on [10]. Study [11] showed that the mechanical properties of silicone were similar to human skin. Medical silicone was used as an imitation of human skin. Silicon material properties were measured by universal material testing machine (LR10KPlus, LLOYD, UK) as shown in Table 1.

Table 1 Mechanical properties of silicon

\begin{tabular}{ccccc}
\hline $\begin{array}{c}\text { Young's modulus } \\
(\mathrm{MPa})\end{array}$ & $\begin{array}{c}\text { Tear strength } \\
(\mathrm{MPa})\end{array}$ & $\begin{array}{c}\text { Breaking } \\
\text { elongation } \\
(\%)\end{array}$ & $\begin{array}{c}\text { Density } \\
(\mathrm{kg} / \mathrm{m} 3)\end{array}$ & $\begin{array}{c}\text { Thickness } \\
(\mathrm{mm})\end{array}$ \\
\hline $3.2-3.6$ & $2.4-4.0$ & $460-500$ & $1100-1200$ & 6.0 \\
\hline
\end{tabular}

Buy medical needles with diameters of $0.5 \mathrm{~mm}, 0.7 \mathrm{~mm}, 0.9 \mathrm{~mm}$ and $1.2 \mathrm{~mm}$ and tip angle of $30^{\circ}$ (Guangzhou Huaxin Trade Co., Ltd.). Grind and polish needle tip angle into $30^{\circ}, 50^{\circ}, 70^{\circ}, 90^{\circ}$ and triangular cone with the diameter $0.7 \mathrm{~mm}$ respectively. The experimental medical needles were shown in Fig. 1.

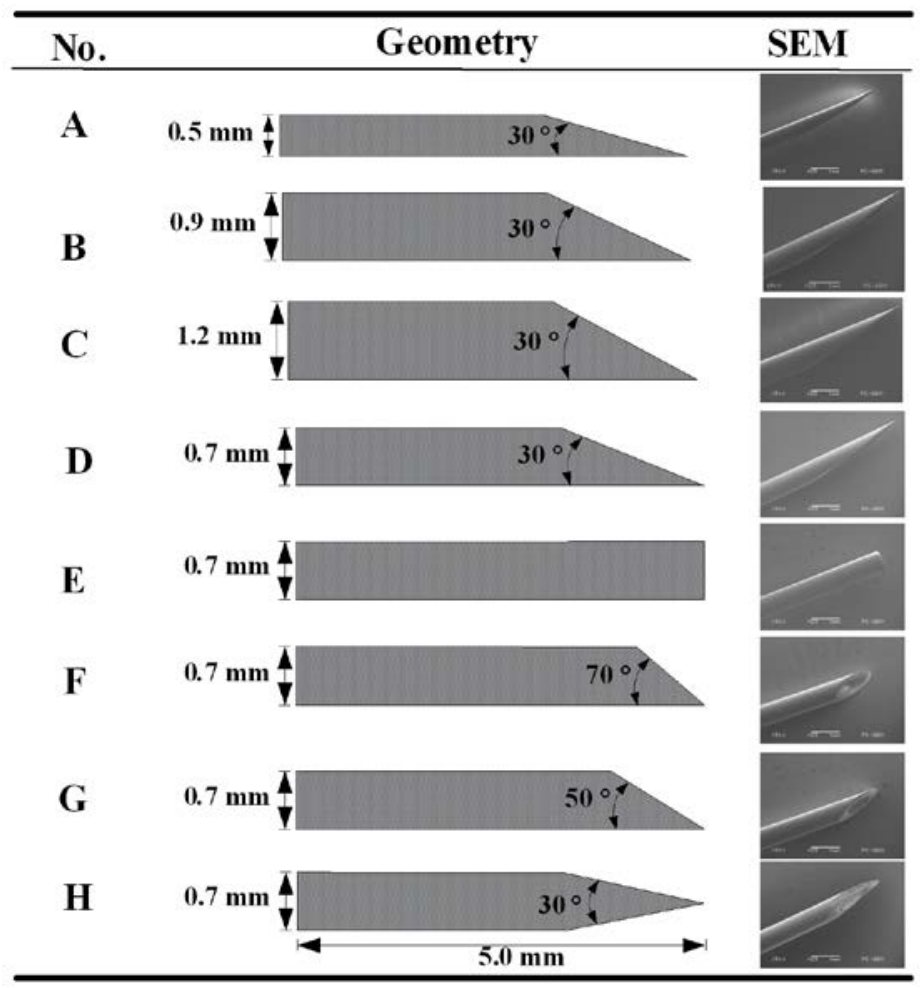

Figure 1 Medical needles

\subsection{Experimental Setup.}

Injection process was tested by self-developed micro-force mechanical loading equipment as shown in Fig. 2. The equipment was mainly consisted of three modules: (1) DC linear motor (M-227.10, Physic Instrument, Germany) with step of $0.01 \mu \mathrm{m}$ and travel range of $10 \mathrm{~mm}$. (2) Micro-force sensor (407A, Aurora Scientific Inc, Canada) with resolution of $20 \mu \mathrm{N}$ and force range of $1000 \mathrm{mN}$. It was assembled with DC motor. (3) Microscope composed of long focal distance objective lens (VH-Z250R, Keyence, Japan) and CCD camera (XC-ST50, Sony, Japan). It could continuously record injection process. The force and displacement could be acquired by LabVIEW program. 

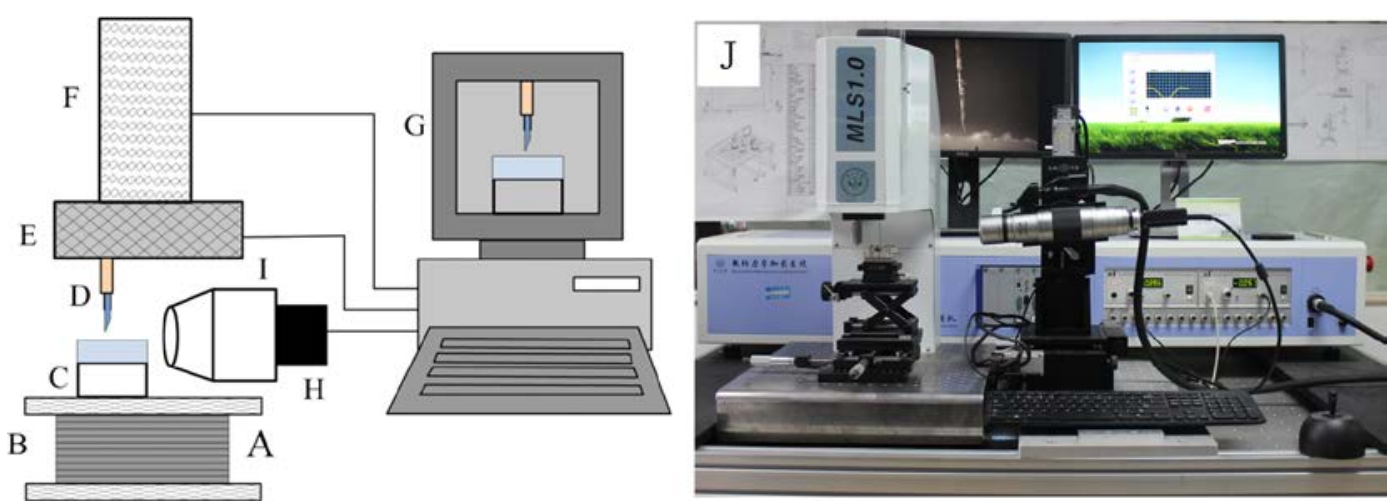

Figure 2 Micro-force mechanical loading equipment: (A) shockproof device; (B) XY axis positioning device; (C) fixture; (D) silicon; (E) micro-force sensor; (F) DC linear motor; (G) computer; (H) CCD;

\subsection{Experimental Procedure.}

(H) len; (J) Loading equipment.

The puncture and pull force test was following: (1) Preparation: fix needle on micro-force sensor and artificial skin silica on fixture; (2) Puncture stage: drive linear motor with needle downward to artificial skin. This loading displacement was $5 \mathrm{~mm}$ and the velocity was $0.25 \mathrm{~mm} / \mathrm{s}, 0.5 \mathrm{~mm} / \mathrm{s}, 0.75$ $\mathrm{mm} / \mathrm{s}$ and $1.0 \mathrm{~mm} / \mathrm{s}$ respectively; (3) Pull stage: drive the linear motor in the reverse direction and pull needle from artificial skin.

\section{Finite Element Analysis}

The Injection process was finite element simulated by Abaqus and its model was shown in Fig. 3. The injection needle was assumed as a rigid material. The needle tip angle was $30^{\circ}$. The silica was meshed using continuum hexahedral elements. It was set as bilinear elastoplastic and strain-failure material [12-13]. The elastic modulus was $2.2 \mathrm{MPa}$, Poisson's ratio was 0.45 , the geometric model diameter was $25 \mathrm{~mm}$ and thickness was $20 \mathrm{~mm}$. The contact type was face penetration [14-16], and Coulomb friction coefficient between the needle and silica was 0.1 [17]. The failure strength of human skin was about $10 \mathrm{MPa}$ [18]. The needle was moved towards silicon with $1 \mathrm{Mpa}$ normal stress. The displacement boundary conditions on the human silica included nodes along the bottom and right edges that were pinned, whereas the top edge was traction free. The calculation was divided into 40 incremental steps and Full Newton-Raphson iterative procedure was adopted as solution method.

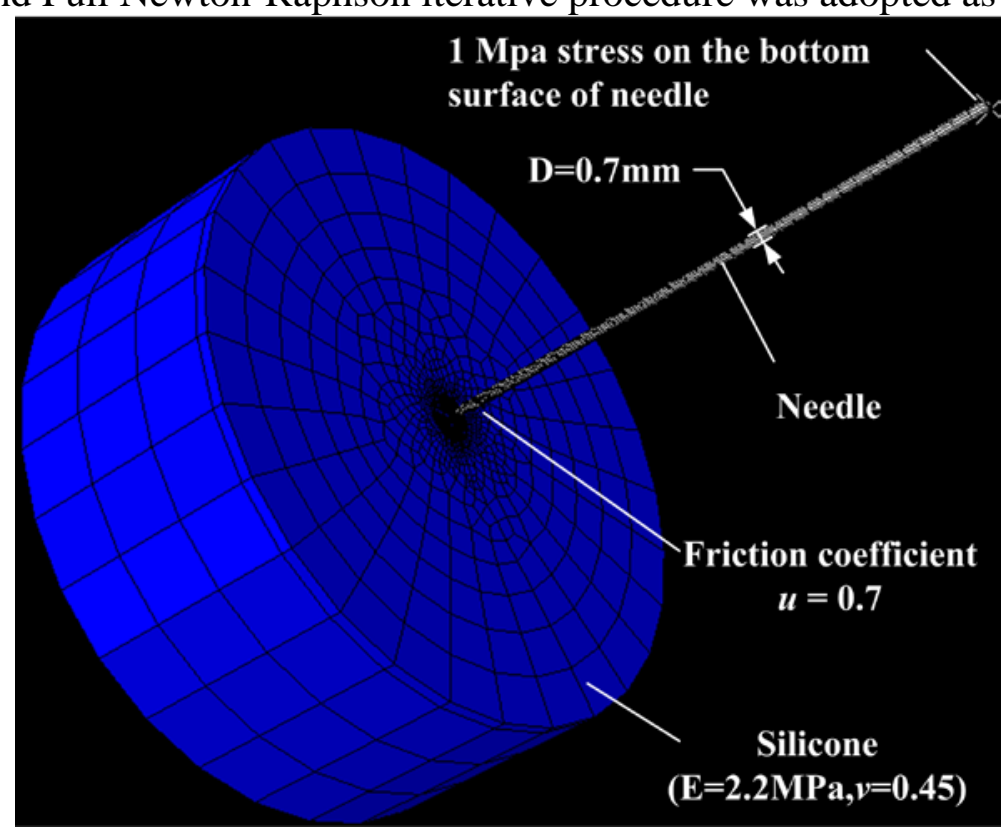

Figure 3 FEM model of medical needle injection process

References are cited in the text just by square brackets [1]. (If square brackets are not available, slashes may be used instead, e.g. /2/.) Two or more references at a time may be put in one set of 
brackets $[3,4]$. The references are to be numbered in the order in which they are cited in the text and are to be listed at the end of the contribution under a heading References, see our example below.

\section{Results and Discussion}

\subsection{Effect of Needle Diameter.}

Fig. 4 showed the effect of diameter on puncture and pull force at the speed of $0.5 \mathrm{~mm} / \mathrm{s}$ and tip angle of $30^{\circ}$. The resistance force increased linearly from zero once the needle touched the artificial skin. The artificial skin down concave deformed under the advancing needle tip during the insertion process. The needle tip initiated a crack that expanded a circumferential hole by stretching and tearing artificial skin, permitting needle insertion into skin. The puncture force reached its maximum value at puncture depth of $4 \mathrm{~mm}$. It was due that the increment of sliding friction between silicon and needle. The needle loading energy was used to tear silicon and overcome the sliding friction. The puncture forces were $308.9 \mathrm{mN}, 319.6 \mathrm{mN}, 369.4 \mathrm{mN}, 423.9 \mathrm{mN}$ at needle diameters of $0.5 \mathrm{~mm}, 0.7 \mathrm{~mm}, 0.9$ $\mathrm{mm}, 1.1 \mathrm{~mm}$ respectively. The pull force decreased gradually during the pull process. The resistance force decreased rapidly once the needle was pulled back. The resistance force decreased to zero and then the compression state of artificial skin on the needle was turned to tensile state. The puncture and pull force decreased with needle diameter under the same puncture depth which was consistent with the research of Okamura et al [19].



Figure 4 Effect of diameter on puncture and pull force at the speed of $0.5 \mathrm{~mm} / \mathrm{s}$ and tip angle of $30^{\circ}$

\subsection{Effect of Needle Diameter.}

Fig. 5 showed the effect of tip angle on puncture and pull force at needle diameter of $0.7 \mathrm{~mm}$ and puncture speed of $0.5 \mathrm{~mm} / \mathrm{s}$. The maximum puncture force varied with needle tip angle. The maximum puncture forces of needle A, B, C, D, and E at $4 \mathrm{~mm}$ puncture depth were $708.3 \mathrm{mN}, 587.1$ $\mathrm{mN}, 523.8 \mathrm{mN}, 307.2 \mathrm{mN}$ and $409.3 \mathrm{mN}$. The maximum puncture force of needle A was 2.21 times of needle A. Therefore, the tip angle greatly influenced the puncture force and D-type needle tip was relatively better. The result agreed with the research of Okamura et al [19] and Webster et al [20]. The needle tip angle should be sharpened to decreased the puncture and pull force if the needle tip stiffness could be guaranteed. 


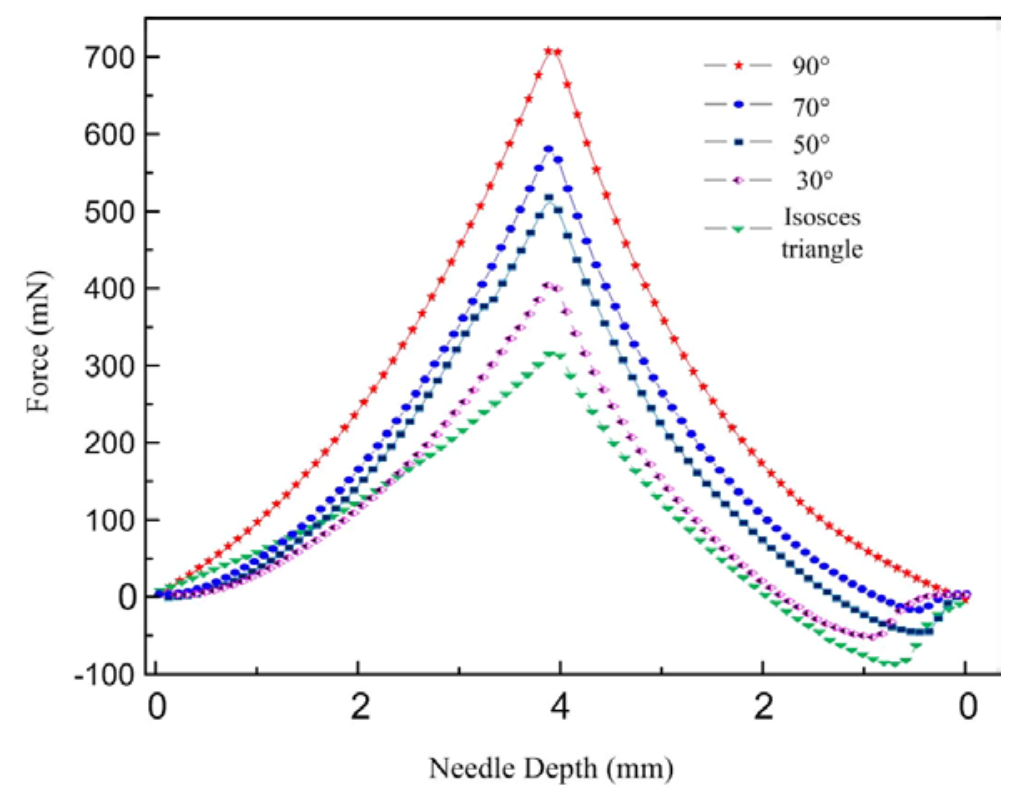

Figure 5 Effect of tip angle on puncture and pull force at the speed of $0.5 \mathrm{~mm} / \mathrm{s}$ and needle diameter of

\subsection{Effect of Speed.}

$0.7 \mathrm{~mm}$

Fig. 6 showed effect of speed on puncture and pull force at the needle diameter of $0.7 \mathrm{~mm}$ and tip angle of $30^{\circ}$. Puncture and pull force of needle increased with its speed. Puncture force at the speed of $0.25,0.5,0.75,1.0 \mathrm{~mm} / \mathrm{s}$ was $290.1 \mathrm{mN}, 307.2 \mathrm{mN}, 319.6 \mathrm{mN}$ and $322.8 \mathrm{mN}$ when the puncture depth was $4 \mathrm{~mm}$. It also indicated that the effect of speed on the puncture force was relatively slight.

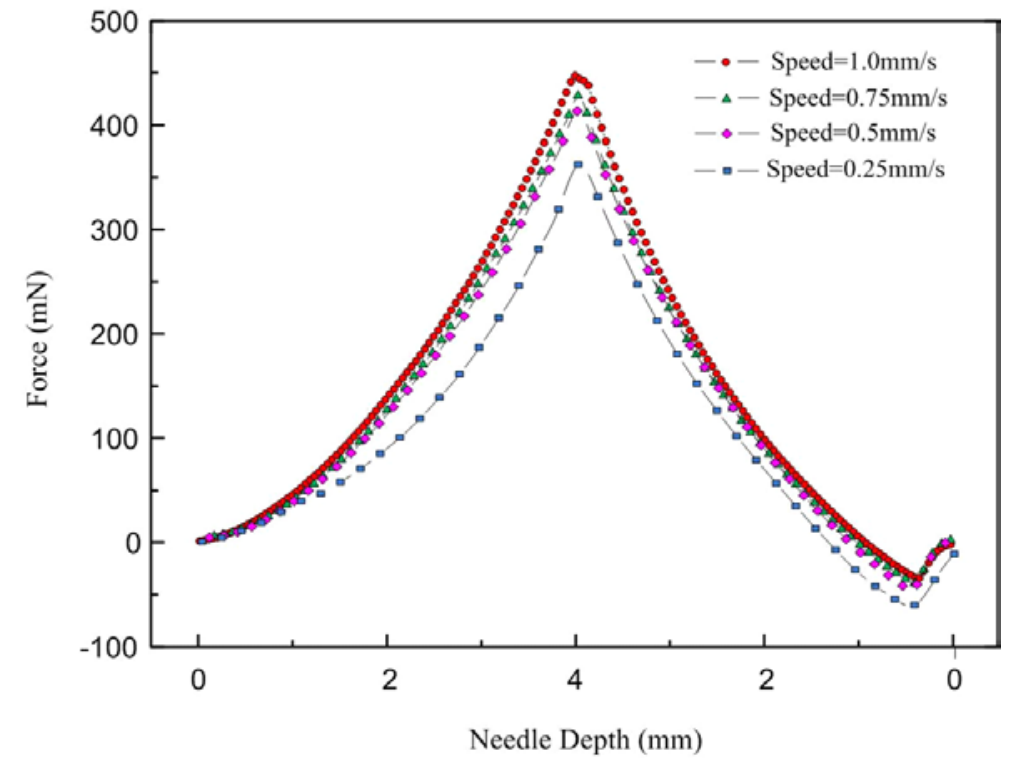

Figure 6 Effect of speed on puncture and pull force at the needle diameter of $0.7 \mathrm{~mm}$ and tip angle of

\subsection{Effect of Speed.}

$30^{\circ}$

Fig. 7 was the stress and strain distribution of needle injection process. Once the needle touched the artificial skin, silica skin initially deformed under the advancing needle tip until a critical load led to human silica puncture. At this critical force, the needle tip initiated a crack that expanded a circumferential hole by stretching and tearing artificial skin, permitting needle tip insertion into skin. The crack faces were wedged open similar to needle insertion into skin. The puncture of artificial skin was planar mode I crack. The needle puncture energy was absorbed by artificial skin during insertion process and led to skin stretching and tearing. As an interaction between needle and artificial skin, resistance increased as the energy was transferred. The puncture stress and strain was maximum value at the needle tip as shown in Fig. 7 (a) and (f). The tip was fully inserted into artificial skin. The strain and stress of artificial skin increased with puncture depth due to the increase of friction between skin 
and needle as shown in Fig. 7 (b) and (g). When puncture depth was $4.0 \mathrm{~mm}$, the puncture stress and strain at needle tip reached its maximum value as shown in Fig. 7 (c) and (h). The stress and strain at needle tip decreased rapidly as the needle was pulled which was due to the decrease of friction between needle and skin. The artificial skin was gradually deformed profiling an up concaved shape as shown in Fig. 7 (d) and (i). The interaction between artificial skin and needle decreased, so the stress and strain decreased around the needle as shown in Fig. 7 (e) and (j).

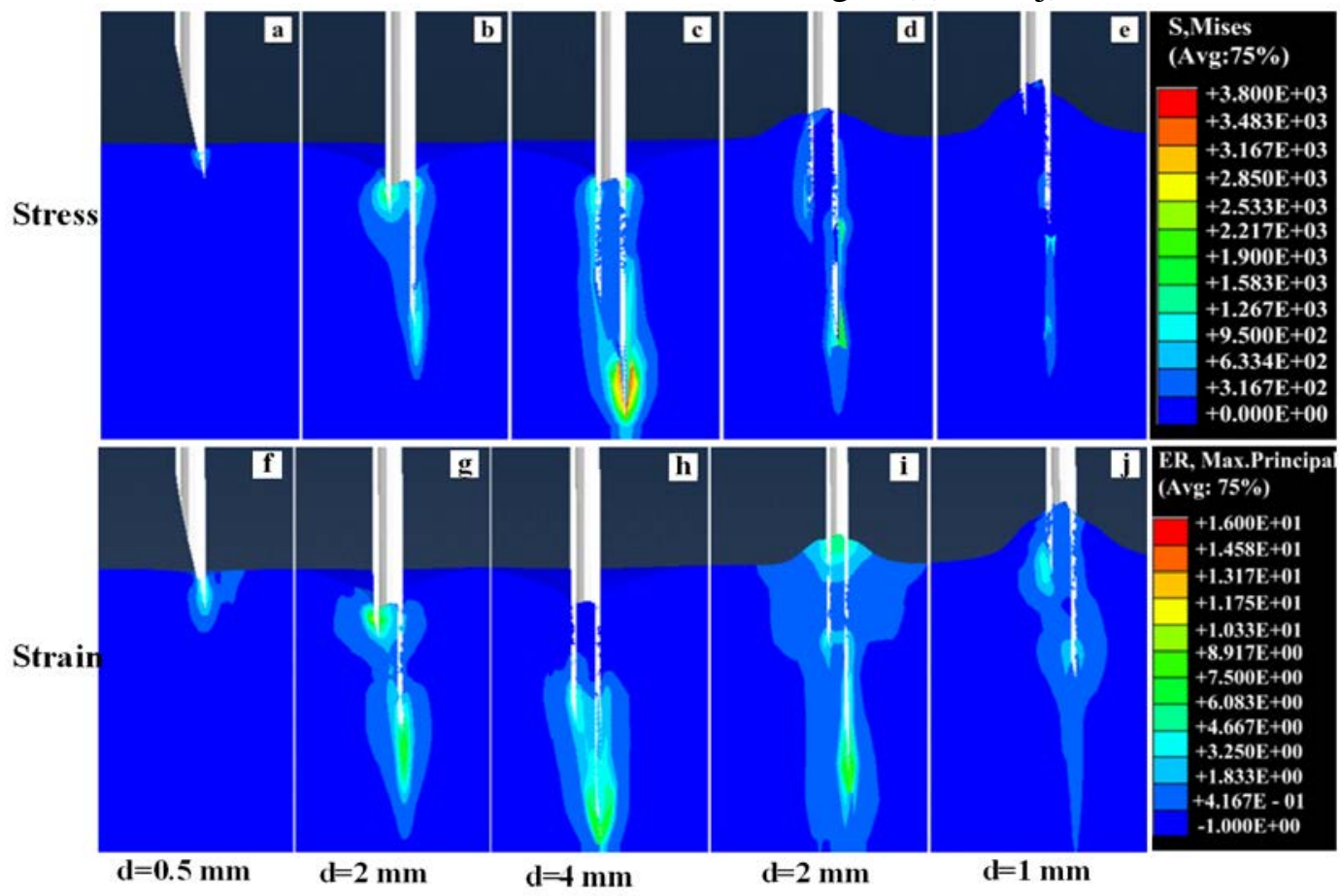

Figure 7 Stress and strain distribution of needle injection process

\section{Conclusions}

Finite element method was proposed to study mechanical properties of medical needle insertion process. Medical needle insertion force at different diameters, needle tip angles and puncture speeds was also investigated. We drew following conclusions: the puncture and pull force decreased with needle diameter under the same puncture depth; the sharpened needle tip angle could decrease the puncture and pull force if the stiffness of needle tip was hard enough; the puncture speed of medical needle slightly affected the puncture force; the puncture of medical needle into skin was mode I crack; the maximum stress and strain was focused on the needle tip.

\section{Acknowledgment}

This research is financially supported by the National Nature Science Foundation of China (Project No. 51205423), Guangdong Innovative Research Team Program (No. 2009010057) and the Fundamental Research Funds for the Central Universities, Sun Yat-sen University (Project No. 13lgpy13).

\section{References}

[1] Zivanovic A, Davies B L. Robotic system for blood sampling[J]. IEEE Transactions on Information Technology in Biomedicine , 2000, 4(1): 8-14.

[2] Yan L, Xin Q, Qian C. Optimize by Experimental Optimization Techniques on the Structure of Corrugated Bionic Needle[J]. Advances in Natural Science, 2010, 3(2)103-112.

[3] Podder T, Clark D, Sherman J, et al. In vivo motion and force measurement of surgical needle 
intervention during prostate brachytherapy [J]. Medical physics, 2006, 33(8): 2915-2922.

[4] O'leary M D, Simone C, Washio T, et al. Robotic needle insertion: Effects of friction and needle geometry[C], IEEE International Conference on Robotics and Automation, 2003(2)1774-1780.

[5] Saito H, Yanai S, Ohta Y, et al. Comparison of vibration waveform and frequency effecting to reduce the needle puncturing force[J]. Proc Japanese Society for Medical and Biological Eng, 2003, 199.

[6] Aoyagi S, Izumi H, Fukuda M. Biodegradable polymer needle with various tip angles and effect of vibration and surface tension on easy insertion [C], Proceedings of the IEEE International Conference on Micro Electro Mechanical Systems, 2007:397-400.

[7] Dimaio S P, Salcudean S E. Needle insertion modeling and simulation[J]. IEEE Transactions on Robotics and Automation, 2003, 19(5): 864-875.

[8] Xin Q, YingChun Q, Yan L, et al. The Insect Fascicle Morphology Research and Bionic Needle Pierced Mechanical Mechanism Analysis [J]. Advances in Natural Science, 2010, 3(2): 57-62.

[9] Maurin B, Barbe L, Bayle B, et al. In vivo study of forces during needle insertions[C], Proceedings of the Scientific Workshop on Medical Robotics, Navigation, and Visualization, 2004: 415-422.

[10] Xu F, Lu T J and Seffen K A. Biothermomechanics of skin tissues[J]. J. Mech. Phys. Solids 2008(56):1852-84.

[11] Shergold O A and Fleck N A. Experimental investigation into the deep penetration of soft solids by sharp and blunt punches, with application to the piercing of skin[J]. J. Biomech. Eng. 2005(127): 838-48

[12] Su J, Zhao W Z, Chen B Z, et al. Establishing finite element contact model of human L1-L5 lumbar segments[J]. Journal of Medical Biomechanics, 2010, 25(3):200-205.

[13] Ibrahim M N, Siswanto W A, Zaidi A. Computational Issues in the Simulation of High Speed Ballistic Impact: A Review[J]. Applied Mechanics and Materials, 2013, 315(7):62-72.

[14] Cheung J T-M, Zhang M, An K-N. Effects of plantar fascia stiffness on the biomechanical responses of the ankle-foot complex[J]. Clinical Biomechanics, 2004, 19(8): 839-846.

[15] Oonishi H, Isha H, Hasegawa T. Mechanical analysis of the human pelvis and its application to the artificial hip joint-by means of the three dimensional finite element method [J]. Journal of biomechanics, 1983, 16(6): 427-444.

[16] Winters J M, Stark L. Estimated mechanical properties of synergistic muscles involved in movements of a variety of human joints[J]. Journal of biomechanics, 1988, 21(12): 1027-1041.

[17] Aoyagi S, Izumi H, Fukuda M. Biodegradable polymer needle with various tip angles and consideration on insertion mechanism of mosquito's proboscis[J]. Sensors and Actuators A: Physical, 2008, 143(1): 20-28.

[18] Ankersen J, Birkbeck AE, Thomson RD, Vanezis P. Puncture resistance and tensile strength of skin simulants. Proc Inst Mech Eng Part H-J Eng Med, 1999, 213(H6):493-501.

[19] Okamura A M, Simone C, O'leary M D. Force modeling for needle insertion into soft tissue[J]. IEEE Transactions on Biomedical Engineering, 2004, 51(10): 1707-1716.

[20] Webster R J, Memisevic J, Okamura A M. Design considerations for robotic needle steering[C], IEEE International Conference on Robotics and Automation, 2005: 3588-3594. 